\section{Hidroxiapatita sintetizada a partir de la cáscara de huevo como potencial sustituto óseo en defectos periodontales y periimplantarios}

\section{Hydroxyapatite synthesized from the eggshell as a potential bone substitute in periodontal and peri-implant defects}

\begin{abstract}
Resumen
La hidroxiapatita sintética es un biomaterial ampliamente utilizado en los procedimientos de regeneración ósea guiada. Objetivo: Sintetizar hidroxiapatita sintética a partir de la cáscara de huevo bajo distintos precipitados de ácido nítrico. Métodos: Estudio experimental que obtuvo $\mathrm{CaCO}_{3}$ a partir de la pulverización de la cáscara de huevo (Gallus gallus). El carbonato fue titulado con tres concentraciones de ácido nítrico (5, 10 y 15\%) y fosfato de amonio. De las mezclas se realizaron pruebas a distintas temperaturas de calcinación $\left(150,600,700,800\right.$ y $\left.900^{\circ} \mathrm{C}\right)$ y tiempos de calcinación $(2,4,8$ y $12 \mathrm{~h})$. Se utilizó el análisis de difracción de rayos $\mathrm{X}$ para caracterizar la presencia de carbonato de calcio e hidroxiapatita. Resultados: De las distintas rutas de síntesis, la vía de precipitación de ácido nítrico por exceso al $15 \%$ más el carbonato de calcio calcinado a $700^{\circ} \mathrm{C}$ durante 8 $\mathrm{h}$ ofreció los resultados más similares a la hidroxiapatita sintética al ser comparado con el patrón estándar comercial. Los picos de intensidad correspondieron a los ángulos 20:20, 26, 33, 34, 40, 48 y 50 Conclusiones: El método de síntesis por precipitación utilizando como sustrato el carbonato de calcio proveniente de la cáscara de huevo más nitrato de calcio y ácido nítrico, evidenció ser adecuado y práctico para la síntesis de hidroxiapatita. Su uso puede ser replicado para los estudios de caracterización y respuesta toxicológica en modelos experimentales pre clínicos.
\end{abstract}

Palabras clave: Cáscara de huevo; Difracción de rayos X; Hidroxiapatita; Precipitación química; Regeneración ósea.

\begin{abstract}
Synthetic hydroxyapatite is a biomaterial widely used in guided bone regeneration procedures. Objective: Synthesize synthetic hydroxyapatite from eggshells under different precipitates of nitric acid. Methods: Experimental study, which obtained $\mathrm{CaCO}_{3}$ from the pulverization of eggshells (Gallus gallus). The carbonate was titrated with three concentrations of nitric acid $(5,10$ and $15 \%)$ and ammonium phosphate. The mixtures were tested at different calcination temperatures $\left(150,600,700,800\right.$ and $\left.900{ }^{\circ} \mathrm{C}\right)$ and calcination times $(2,4,8$ and $12 \mathrm{~h})$. X-ray diffraction analysis was used to characterize the presence of calcium carbonate and hydroxyapatite. Results: Of the different routes of synthesis, the route of precipitation of nitric acid by excess to $15 \%$ plus calcined calcium carbonate at $700{ }^{\circ} \mathrm{C}$ during $8 \mathrm{~h}$ offered more equivalent results to the synthetic hydroxyapatite when compared with the commercial standard. The peaks of intensity corresponded to the angles 20:20, 26, 33, 34, 40, 48 and 50. Conclusions: The method
\end{abstract}

\section{Artículo Original}

Isabel Camacho Díaz ${ }^{1, a}$, Melissa Vela Rodríguez ${ }^{1, a}$, Ludolfo Villanueva Aburto ${ }^{1, a}$ Andrés Borja Villanueva ${ }^{1, b}$, Fred Montalvo Amanca $^{2, c}$, Justiniano Quispe Marcatoma ${ }^{2, d}$

${ }^{1}$ Universidad Privada Juan Pablo II, Facultad de Ciencias de la Salud. Lima, Perú.

2 Universidad Nacional Mayor de San Marcos, Facultad de Física. Centro de Investigaciones Tecnológicas, Biomédicas y Medioambientales. Lima, Perú.

a Estudiante.

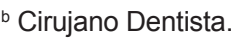

c Bachiller en ciencias físicas.

d Doctor en ciencias físicas.

\section{Correspondencia:}

Isabel Camacho Díaz

Correo electrónico: vicamacho20@hotmail.com Calle Ricardo Palma \#136 Collique 5ta zona.

Comas. Perú.

\section{Coautores:}

Melissa Vela Rodríguez camevelarodríguez@hotmail.com Ludolfo Villaneva Aburto ludvilla123@hotmail.com Andrés Borja Villanueva abv1979@gmail.com Justiniamo Quispe Marcatoma jquispem@citbm.pe Fred Montalvo Amanca bracstam@gmail.com

Conflicto de intereses: Los autores declaran no tener conflictos de interés.

Fuente de financiamiento: Autofinanciado

Fecha de recepción: 10/08/18

Fecha de aceptación: 07/10/18 
of synthesis by precipitation, using calcium carbonate from eggshells as substrate plus calcium nitrate and nitric acid; proved to be adequate and practical for the synthesis of hydroxyapatite. Its use can be replicated for toxicological characterization and response studies in pre-clinical experimental models.

Keywords: Bone regeneration; Chemical precipitation; Egg shell; Hydroxyapatite; X-Ray diffraction.

\section{Introducción}

Los defectos óseos bucomaxilofaciales pueden ser tratados a través de la distracción osteogénica y los procedimientos de regeneración ósea guiada (ROG). La ROG a su vez puede utilizar sustitutos óseos autógenos, alógenos, xenógenos y sintéticos. Excluyendo a los sustitutos autógenos ninguno ha demostrado regenerar el tejido óseo principalmente por las interrogantes referidas a su estabilidad mecánica, biocompatibilidad in vivo a largo plazo y biodegrabilidad ${ }^{1}$. Actualmente se desarrollan alternativas a los sustitutos óseos cuyas propiedades sean mejoradas constantemente.

En el campo de la Periodoncia e Implantología oral los sustitutos óseos más utilizados corresponden a cerámicas de fosfato de calcio sintéticos a base de hidroxiapatita (HAp) o fosfato $\beta$-tricálcico. La hidroxiapatita sintética es un tipo de sustituto óseo compuesto de fosfato y calcio con una estructura similar al componente inorgánico del tejido óseo humano. Sus componentes permiten que provea de estabilidad y dureza a la estructura cristalina ${ }^{2}$. Su biocompatibilidad ha sido demostrada por la interacción del $\mathrm{Ca}^{+2}$ y el $\mathrm{PO}^{-3}$ dentro de las funciones celulares y fisiológicas ${ }^{3}$, además de ser bioactivo al degradarse sin alterar el proceso de cicatrización del tejido óseo ${ }^{4}$; además que su biodegrabilidad es dependiente de su composición y propiedades físico químicas ${ }^{5,6}$.

Actualmente existe un gran interés en sintetizar biocerámicas que presenten una morfología que facilite e induzca el crecimiento del tejido óseo así como facilitar la oseointegración del implante dental ${ }^{1}$. De esta forma el interés por obtener HAp sintética porosa está principalmente motivado por el potencial de restauración en vascularidad y la completa penetración del tejido óseo en el sitio de cicatrización ${ }^{7}$.

Algunos estudios consideran la posibilidad de sintetizar HAp a partir de una cáscara de huevo, esto debido a que es una estructura a base de $\mathrm{CaCO}_{3}(94 \%)$, carbonato de magnesio $(1 \%)$, fosfato de calcio $(1 \%)$ y trazas de otros minerales $(4 \%)^{8}$ que puede ser utilizada para sintetizar HAp carbonatada ${ }^{9,10}$; sus usos como material pulverizado se han realizado como fertilizantes, producción de suplementos nutricionales y como materiales de relleno óseo ${ }^{11}$. El uso de la HAp obtenida a partir de la cáscara de huevo representa un biomaterial alternativo para la práctica clínica además de ser más económico tanto para la empresa manufacturera como para los pacientes?

De los distintos métodos de obtención de la HAp se ha puesto interés en la generación de apatitas precipitadas en soluciones acuosas; esto debido a que la precipitación entre los $37-60{ }^{\circ} \mathrm{C}$ se encuentra pobremente cristalizada y se asemeja a la apatita natural ${ }^{11}$. Su síntesis a partir de precipitados busca utilizar al calcio como mediador durante el remodelado óseo y estimulador de células osteoprogenitoras así como sustrato para producir señales quimiotácticas ${ }^{12}$ y los fosfatos como elementos que influyen en la mineralización de la matriz ósea pero en exceso puede provocar apoptosis celular ${ }^{13}$. En la presente investigación se buscó obtener hidroxiapatita a partir de la cáscara de huevo según el método de precipitados.

\section{Métodos}

Obtención de carbonato de calcio a partir de cáscara de huevo. La síntesis de HAp nanocristalina implicó la preparación del precipitado paso a paso. Se utilizó la técnica de precipitación como técnica base para preparar HAp. Se utilizó como sustrato la cáscara del huevo de gallina (Gallus gallus) debido a su elevado componente de calcita $\left(95 \%\right.$ de $\left.\mathrm{CaCO}_{3}\right)$.

Se obtuvo $6 \mathrm{Kg}$ de huevos de los cuales se extrajo sus sellos, yema y clara para solo quedarnos con las cáscaras. Se utilizó $600 \mathrm{~g}$ de cáscara que fueron procesadas manualmente y limpiadas en una solución de hipoclorito de sodio $(350 \mathrm{ml})$ y agua desionizada $(350 \mathrm{ml})$ que reposaron en una campana de extracción (Trimming Tech $\AA$, Italia) por un total de 36 horas a temperatura ambiente para luego ser secadas a $70^{\circ} \mathrm{C}$ por 30 minutos en una estufa (Bautermic S.A ${ }^{\circledR}$, España).

Luego se procedió a su molienda manual a través de un molino planetario (Laval Lab ${ }^{\circledR}$, Canadá) para obtener partículas menores a $100 \mu \mathrm{m}$ con la ayuda de un tamizador de $90 \mu \mathrm{m}$ (Tamiz 8“" No 170, ATM $®$, U.S.A). El polvo fue sometido al difractor de rayos $\mathrm{X}$ para comprobar la presencia de $\mathrm{CaCO}_{3}$. El $\mathrm{CaCO}_{3}$ obtenido se tituló con tres soluciones de ácido nítrico por exceso bajo distintas condiciones de neutralidad del $\mathrm{pH}$.

Síntesis de hidroxiapatita por exceso de ácido nítrico. Del polvo obtenido de la cáscara de huevo, se seleccionó 15 g para dividirlo en tres muestras de $5 \mathrm{~g}$ cada una que serían combinadas con tres concentraciones de ácido nítrico (5, 10 y 15\%). En las tres muestras el procedimiento fue el mismo con la diferencia en las proporciones de las mezclas.

Muestra 1: para la síntesis de HAp de la muestra 1 se utilizó 1,43 g del carbonato de calcio, 1,34 ml de ácido nítrico (5\%), 0,6 g de fosfato de amonio y $8,9 \mathrm{ml} \mathrm{de}$ agua. La solución se llevó a la hornilla de agitación magnética y se programó el agitador a $500 \mathrm{rpm}$ (Dinko Instruments D-12 ®; España), agregando progresivamente el ácido nítrico hasta llegar al punto que no haya efervescencia, en total fue agregado $10 \mathrm{ml}$ de ácido nítrico. A 
esta solución le fue agregada nitrato de calcio a un $\mathrm{pH}$ de 10,5 elevada con nitrato de amonio.

En una segunda solución se preparó $0,6 \mathrm{~g}$ de fosfato de amonio con 12,4 $\mathrm{ml} \mathrm{H}_{2} \mathrm{O}$. Elevada a un $\mathrm{pH}$ de 10,5 con 200 gotas de hidróxido de amonio. Esta segunda solución fue vertida en una pera de decantación para a continuación comenzar el goteo sobre el nitrato de calcio. Terminado el goteo la mezcla estuvo en agitación continua por $600 \mathrm{rpm}$ durante 2 horas.

La muestra resultante fue secada en la estufa (Bautermic S.A $\circledast$, España) por cuatro horas a $150^{\circ} \mathrm{C}$ y luego se calcinó por $8 \mathrm{~h} \mathrm{a} 700^{\circ} \mathrm{C}$ para su posterior análisis químico.

Muestra 2: el procedimiento fue similar a la muestra 1 con la diferencia de las cantidades a utilizar en la primera fase: se utilizó $1,43 \mathrm{~g} \mathrm{de} \mathrm{CaCo}_{3}$, más $1,4 \mathrm{ml}$ de $\mathrm{HNO}_{3}$ $(10 \%)$ con un exceso de 7,4 ml. Se programó el agitador a $600 \mathrm{rpm}$, agregando progresivamente el ácido nítrico hasta llegar al punto que no haya efervescencia, en total fue agregado $7,4 \mathrm{ml}$.

Al nitrato de calcio ya preparado fue agregado $8,9 \mathrm{ml}$ $\mathrm{H}_{2} \mathrm{O}$ y mezclado en el agitador magnético. Fue elevada a un $\mathrm{pH}$ de 10,5 con 38 gotas hidróxido de amonio.

Muestra 3: el procedimiento fue similar a la muestra 1 con la diferencia de las cantidades a utilizar en la primera fase: se utilizó $1,43 \mathrm{~g}$ de $\mathrm{CaCO}_{3}$, más $1,46 \mathrm{ml}$ de $\mathrm{HNO}_{3}(15 \%)$ con un exceso de $18 \mathrm{ml}$. Se programó el agitador a $600 \mathrm{rpm}$, agregando progresivamente el ácido nítrico hasta llegar al punto que no haya efervescencia,
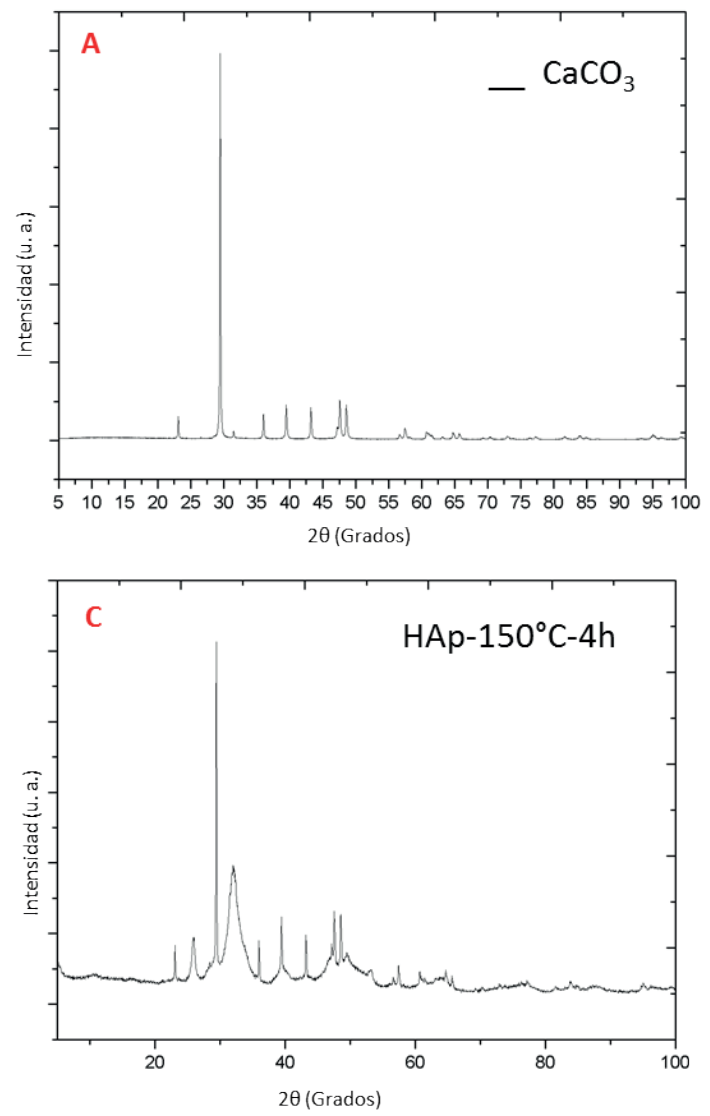

en total fue agregado $18 \mathrm{ml}$. Al nitrato de calcio ya preparado fue agregado $8,9 \mathrm{ml} \mathrm{H}_{2} \mathrm{O}$ y mezclado en el agitador magnético. Fue elevada a un $\mathrm{pH}$ de 10,5 con 38 gotas hidróxido de amonio.

Caracterización de la hidroxiapatita. Para la identificación de los elementos obtenidos en cada muestra se utilizó el análisis de difracción de rayos X (DRX). Se utilizó un difractómetro de rayos X Siemens D-5000 $\mathrm{y}$ tubo de rayos $\mathrm{X}$ con ánodo de cobalto (Co $\mathrm{K}$ a 1,2 emission, $\lambda=1,7903 \mathrm{~A}$ ). Se obtuvieron los difractogramas de las tres muestras de carbonato de calcio mezclada con tres concentraciones de ácido nítrico (5, 10 y 15\%).

La técnica de DRX se aplicó para estudiar las características estructurales de las muestras sintetizadas en diferentes valores de $\mathrm{pH}$. El objetivo final del estudio fue determinar la composición de fase y el rango de la estabilidad de temperatura para los materiales obtenidos según cada muestra.

\section{Resultados}

Se identificó los productos obtenidos a partir del análisis de difracción de rayos $\mathrm{X}$ con las caras cristalinas de cada muestra.

En la muestra obtenida del polvo proveniente de la cáscara de huevo se identificó los picos indicadores de $\mathrm{CaCO}_{3}$ siendo mayores en el ángulo 20: 29 (Figura 1A) mientras que las distintas pruebas de obtención de la HAp en las tres muestras a los $150^{\circ} \mathrm{C}$ no evidenciaron la presencia de la misma (Figuras 1B, C y D).
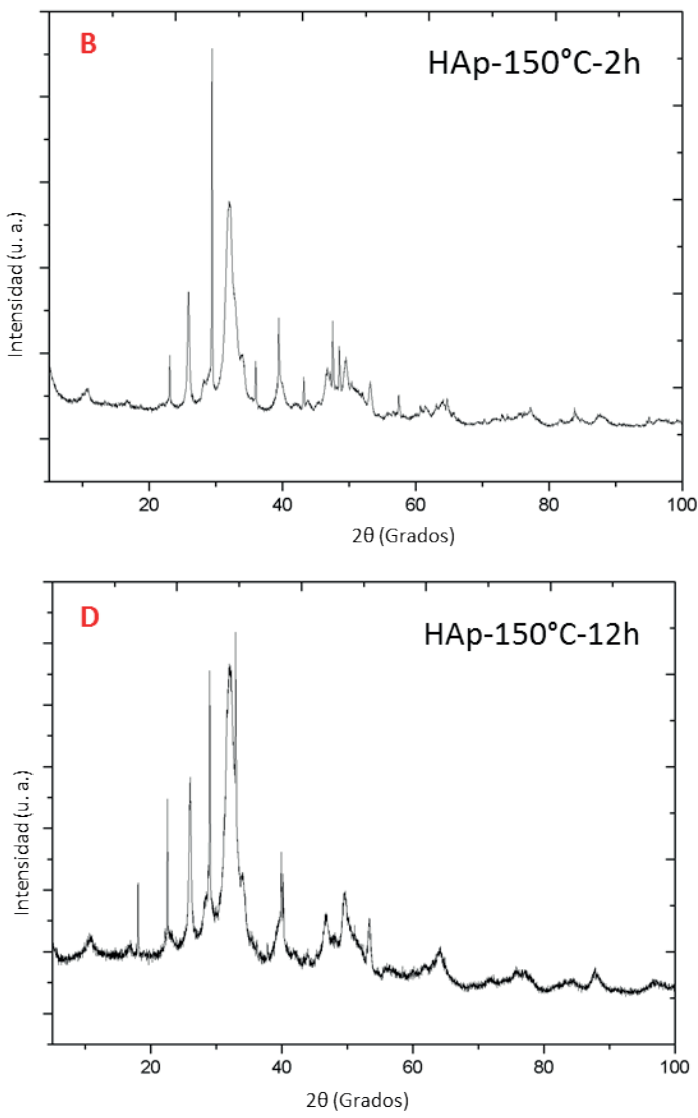

Figura 1. A. Presencia de carbonato de calcio en el polvo proveniente de la cáscara de huevo molido. B. Producto obtenido luego de $2 \mathrm{~h}$ de calentamiento. C. Luego de $4 \mathrm{~h}$ de calentamiento. D. Luego de $12 \mathrm{~h}$ ninguna muestra evidenció similitud a la hidroxiapatita 

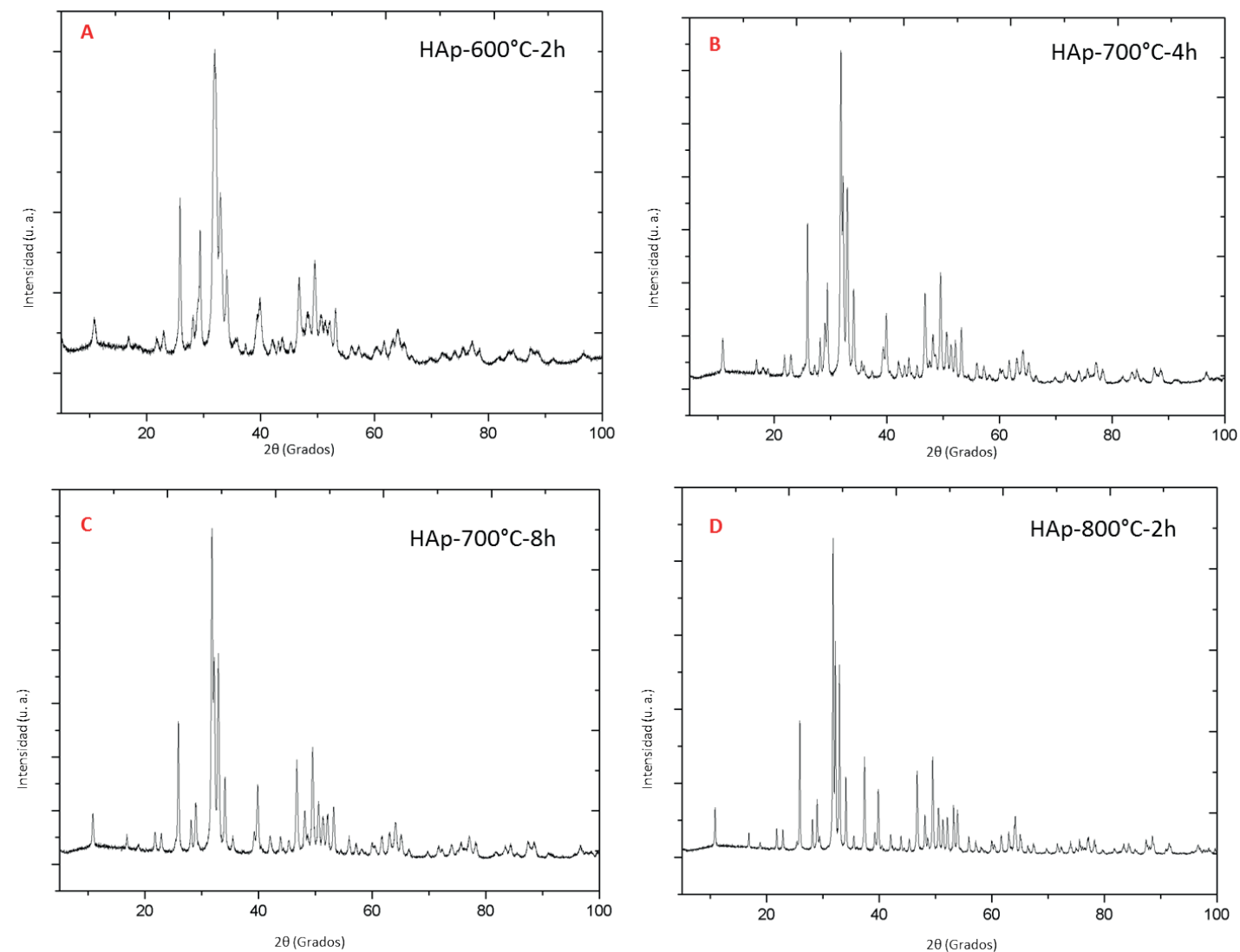

Figura 2. A. Intento de obtención a los $600^{\circ} \mathrm{C}$ al cabo de $2 \mathrm{~h}$. B. Intento a los $700{ }^{\circ} \mathrm{C}$ durante $4 \mathrm{~h}$ de calentamiento. C. A los $700{ }^{\circ} \mathrm{C}$ se presentó mayor similitud a la HAp. D. A los $800^{\circ} \mathrm{C}$ al cabo de $2 \mathrm{~h}$

El aumento de temperatura a $600{ }^{\circ} \mathrm{C}$ evidenció cercanía de obtención de HAp; sin embargo, aún no se logró el ideal (Figuras 2A, B y C).

A una temperatura de $700{ }^{\circ} \mathrm{C}$ se logró que la muestra 3 presentará los picos más importantes de la cáscara de huevo con respecto al estándar de calcita (International Centre for Diffraction Data). Los picos corresponden a los ángulos 20: 26, 33, 34, 40, 48 y 50 (Figura 3).

\section{Discusión}

Los biomateriales fueron desarrollados con la finalidad de reemplazar la forma y función de los tejidos humanos perdidos. De esta forma los defectos óseos buco maxilofaciales pueden ser reparados y regenerados a través del accionar de sustitutos óseos autógenos, alógenos, xenógenos y sintéticos.

El sustituto óseo xenogénico bovino ha sido el biomaterial más utilizado debido a su bajo costo y facilidad de obtención; sin embargo, el riesgo de infección cruzada, reacciones inmunológicas e irritación de la zona intervenida limitan su uso y efectividad ${ }^{14}$. Los sustitutos sintéticos como la hidroxiapatita $\left(\mathrm{Ca}_{10}\left(\mathrm{PO}_{4}\right)_{6}(\mathrm{OH})_{2}\right)$, asemejan a la estructura inorgánica del hueso y se presenta como un biomaterial osteoconductivo ${ }^{15}$. De esta forma, la investigación de nuevos biomateriales y métodos de síntesis que permitan mimetizar la arquitectura del hueso trabecular debe ser una línea de investigación.
La síntesis de la hidroxiapatita en el presente estudio se basó en la muestra de $\mathrm{CaCO}_{3}$ obtenida a partir de la cáscara de huevo, este modelo ha sido planteado en múltiples publicaciones debido al alto contenido de calcita que se presenta ${ }^{16,17}$.

El uso del difractor de rayos $\mathrm{X}$ nos permitió caracterizar los compuestos a medida que se iban realizando las combinaciones según temperatura y tiempo. Los picos de intensidad y la confirmación de la presencia de hidroxiapatita se lograron a una temperatura de calcinación de $700{ }^{\circ} \mathrm{C}$ durante $8 \mathrm{~h}$ y bajo una mezcla de saturación de $\mathrm{HNO}_{3}$ al $15 \%$, el difractor confirmó su presencia principalmente con el aumento en la intensidad en el ángulo 20: 20,99 como indicador de HAp sintetizada a partir de la cáscara de huevo ${ }^{9,18}$.

Se conoce que la HAp puede adherirse directamente a los tejidos humanos y promover su crecimiento. Por lo que su potencial uso como un material poroso y sintético permitiría reproducir mejor las características del hueso a reparar ${ }^{19-21}$. Sin embargo, la HAp sintética presenta el inconveniente de presentar una baja resistencia mecánica por lo que en pocas situaciones es utilizada de forma adherida al implante dental y se prefiere su uso como sustituto óseo en defectos periodontales, regiones adyacentes a implantes y defectos de huesos largos ${ }^{15}$.

La HAp ha sido obtenida a partir de varios métodos (sol-gel 22, químico-húmedo en solución acuosa, hidrotermal ${ }^{23}$, deposición térmica, reacción en estado 


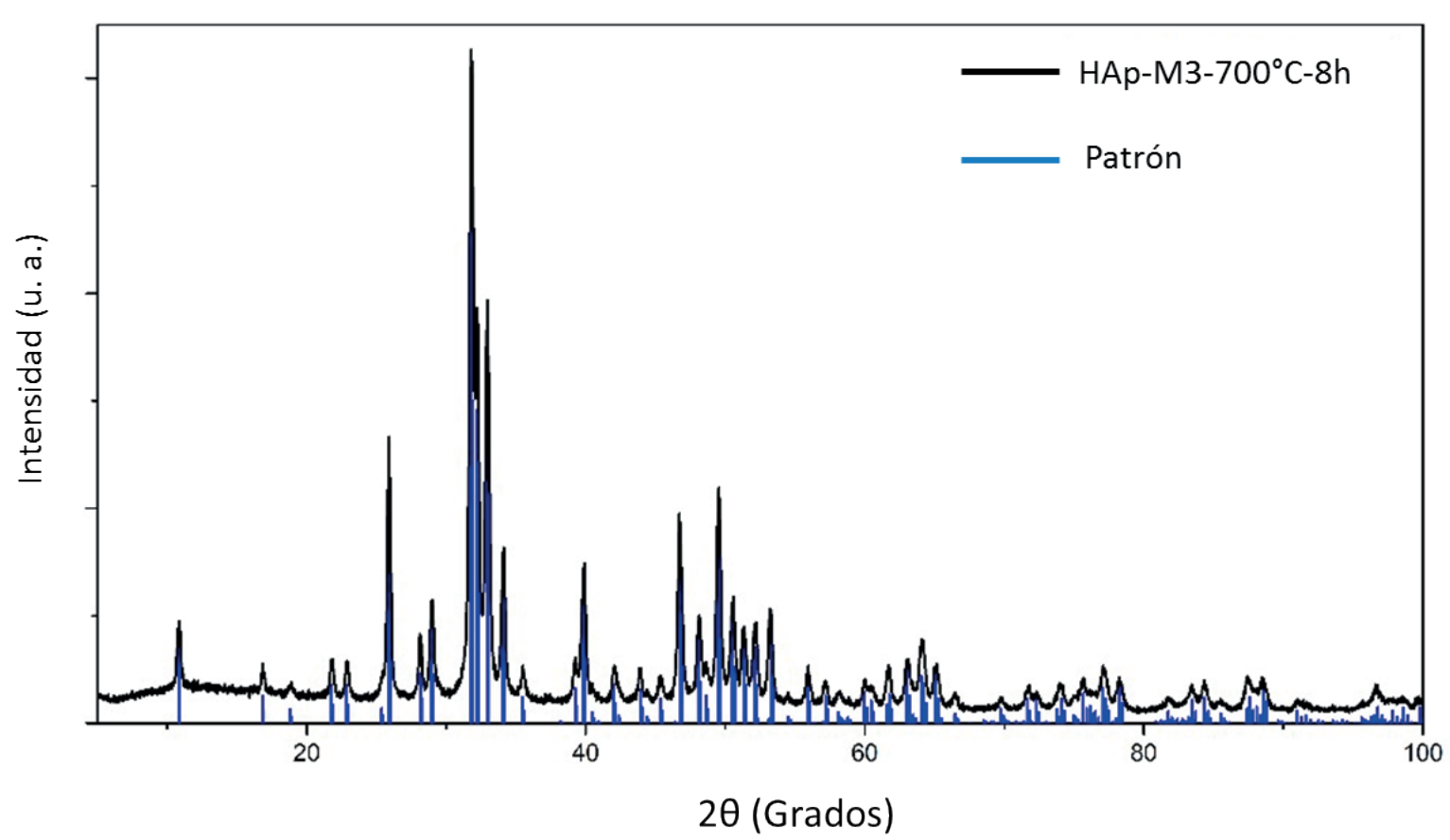

Figura 3. Intensidad de la hidroxiapatita obtenida con la tercera muestra $\left(15 \%\right.$ de $\left.\mathrm{HNO}_{3}\right)$ a $700{ }^{\circ} \mathrm{C}$ durante $8 \mathrm{~h}$ de calentamiento comparada con el patrón estándar

sólido ${ }^{24}$ y la precipitación continua. Obtener HAp a través del método de precipitación continua ofrece las ventajas de no requerir un elevado costo además de ser un método simple; hemos de mencionar que el control del $\mathrm{pH}$, temperatura y orden de mezcla de los reactivos son variables influyentes en la síntesis. Nuestro estudio controló el pH a 10,5 utilizando hidróxido de amonio y nitrato de amonio, esto debido a que valores inferiores, García y cols..$^{25}$ y Sequeda y cols. ${ }^{26}$, han reportado que tiende a formar fosfatos de calcio.

La velocidad de adición de los reactivos se mantuvo entre 1 a $2,5 \mathrm{ml} / \mathrm{min}$ esto para favorecer la nucleación de la HAp; Sequeda y cols. ${ }^{26}$ recomiendan que sea entre 1 a $5 \mathrm{ml} / \mathrm{min}$ y añade que mayores flujos incrementa la formación de especies de fosfato de calcio amorfo ${ }^{27}$. La adición de $\mathrm{HNO}_{3}$ en una mayor proporción (15\%) combinado con el $\mathrm{Ca}\left(\mathrm{OH}_{2}\right)$ permitió que la especie $\left(\mathrm{PO}_{4}\right)^{-3}$ no se encuentre en una concentración suficiente para formar una HAp estequiométrica y se mantenga la proporción de $\mathrm{Ca} / \mathrm{P}=1,67^{28}$.

Los primeros difractogramas realizados a temperaturas de calcinación por debajo de los $700{ }^{\circ} \mathrm{C}$ evidenciaron picos estrechos y de baja intensidad, un indicador claro de que no existía buena cristalinidad y nos encontrábamos lejos del ideal. Acorde al estándar planteado por el "International Centre for Diffraction Data" ${ }^{29}$ se encontró similitud con la HAp a una temperatura de calcinación de $700{ }^{\circ} \mathrm{C}$.

La presente investigación debe ser complementada con un análisis del tamaño de los granos obtenidos, su relación molar de $\mathrm{Ca} / \mathrm{P}$, nivel de pureza y cristalinidad así como detectar la presencia de fases amorfas. Recomendamos estudios más detallados sobre la caracterización de la hidroxiapatita a partir de este método de síntesis.
Una vez alcanzado el ideal de la hidroxiapatita químicamente debe ser utilizada como andamiaje para el crecimiento de células osteoprogenitoras en modelos experimentales preclínicos como la mandíbula de conejo o cobayos.

Concluimos que se pudo sintetizar HAp a partir del $\mathrm{CaCO}_{3}$ obtenido de la cáscara de huevo por el método de precipitación húmeda utilizando titulaciones con ácido nítrico por exceso como disolvente y agente quelante al ser calentado a una temperatura de $700{ }^{\circ} \mathrm{C}$ durante 8 horas, siendo este método sencillo y no acarrea elevados costos.

\section{Agradecimientos}

Al Centro de Investigaciones Tecnológicas, Biomédicas y Medioambientales de la Facultad de Física de la Universidad Nacional Mayor de San Marcos por el apoyo en el trabajo de laboratorio y caracterización de las muestras. Al Dr. Víctor Peña Rodríguez por los permisos otorgados y la orientación en la ejecución del estudio.

\section{Referencias bibliográficas}

1. Tadic D, Beckmann F, Schwarz K, Epple M. A novel method to produce hydroxyapatite objects with interconnecting porosity that avoids sintering. Biomaterials. 2004;25:3335-40.

2. Chai YC, Carlier A, Bolander J, Roberts SJ, Geris L, Schrooten J, et al. Current views on calcium phosphate osteogenicity and the translation into effective bone regeneration strategies. Acta Biomater. 2012;8(11):3876-87.

3. Barrère F, van Blitterswijk CA, de Groot K. Bone regeneration: molecular and cellular interactions with calcium phosphate ceramics. Int J Nanomedicine. 2006;1(3):317-32. 
4. Salinas AJ, Vallet-Regí M. Bioactive ceramics: from bone grafts to tissue engineering. RSC Adv. 2013;3(28):1111631.

5. Ewers R, Goriwoda W, Schopper C, Moser D, Spassova E. Histologic findings at augmented bone areas supplied with two different bone substitute materials combined with sinus floor lifting. Clin Oral Implants Res. 2004;15(1):96-100.

6. Blokhuis TJ, Termaat MF, den Boer FC, Patka P, Bakker FC, Haarman HJ. Properties of calcium phosphate ceramics in relation to their in vivo behavior. J Trauma. 2000;48(1):179-86.

7. Hing K, Best S, Bonfield W. Characterization of porous hydroxyapatite. J Mater Sci Mater Med. 1999; 10(3):135-45.

8. Thapon J, Bourgeois C. L'oeuf et les ovoproduits. 1era ed. París: Lavoisier Tee Doc; 1994.

9. Rivera-Muñoz E, Curiel R, Rodríguez R. Selectivity in the hydroxyapatite synthesis from eggshell using different termal treatments. Mater Res Innov. 2003;7(2):8590 .

10. Prabakaran K, Balamurugan A, Rajeswari S. Development of calcium phosphate based apatite from hen's eggshells. B Mater Sci. 2005;28(2):115-9.

11. Acevedo-Dávila J, López-Cuevas J, Vargas-Gutiérrez G, Rendón-Ángeles J, Méndez-Nonell J. Chemical synthesis of bone-like carbonate hydroxyapatite from hen eggshells and its characterization. J Spanish Ceramic Glass. 2007;46:225-31.

12. Dvorak MM, Siddiqua A, Ward DT. Physiological changes in extracellular calcium concentration directly control osteoblast function in the absence of calciotropic hormones. Proc Natl Acad Sci USA. 2004;101(14):5140-5.

13. Liu YK, Lu QZ, Pei R. The effect of extracellular calcium and inorganic phosphate on the growth and osteogenic differentiation of mesenchymal stem cells in vitro: implication for bone tissue engineering. J Biomed Mater. 2009;4(2):025004. doi: 10.1088/1748$6041 / 4 / 2 / 025004$.

14. Gil-Albarova J, Garrido-Lahiguera R, Salinas AJ, Román J, Bueno-Lozano AL, Gil-Albarova R, et al. The in vivo performance of a sol-gel glass and a glass-ceramic in the treatment of limited bone defects. Biomaterials. 2004;25(19):4639-45.

15. Vallet-Regí M. Revisiting ceramics or medical applications. Dalton Trans. 2006;28(44):5211-20.

16. Nakano T, Ikawa N,Ozimek L. Chemical composition of chicken eggshell and shell membranes. Poult Sci. 2003;82(3):510-4.
17. Chien YC, Hincke MT, McKee MD. Avian eggshell structure and osteopontin. Cells Tissues Organs. 2009;189(1-4):38-43.

18. Ślósarczyk A, Paszkiewicz Z, Paluszkiewcz C. FTIR and XRD evaluation of carbonated hydroxyapatite powders synthesized by wet methods. J Mol Struct. 2005;744:65761.

19. Sigmund WM, Bell NS, Bergstrom L. Novel powder-processing methods for advanced ceramics. J Am Ceram Soc. 2000;7:1557.

20. Prado da Silva MH, Lemos AF, Gibson IR, Ferreira JM, Santos JD. Porous glass reinforced hydroxyapatite materials produced with different organic additives. J Non-Crystall Solids. 2002;304:286.

21. Madhavi S, FerrarisC, White TJ. Synthesis and crystallization of macroporous Hydroxyapatite. J Solid State Chem. 2005;178:2838-45.

22. Kivrak N, Tas AC. Synthesis of calcium hydroxyapatite-tricalcium phosphate composite bioceramic powders and their sintering behavior. J Am Ceram Soc. 1998;81:2245-52.

23. Sivakumar M, Kumar TSS, Shantha KL, Rao KP. Development of hydroxyapatite derived from Indian coral. Biomaterials. 1996;7:1709-14.

24. Tadic D, Epple M. Mechanically stable implants of synthetic bone mineral by cold isostatic pressing. Biomaterials. 2003;24:4565-72.

25. García C, Páucar C, Gaviria J. Study of some parameters that determine the synthesis of hydroxyapatite by the precipitation route. Dyna Rev Fac Nac Minas. [online]. 2006;73(148). [cited 2018-10-10], pp.9-15. Available from: <http://www.scielo.org.co/scielo.php?script=sci_ arttext\&pid=S0012-73532006000100002\&lng=en\&n$\mathrm{rm}=\mathrm{iso}>$. ISSN 0012-7353.

26. Sequeda LG, Díaz JM, Gutiérrez SJ, Perdomo SJ, Gómez OL. Obtención de hidroxiapatita sintética por tres métodos diferentes y su caracterización para ser utilizada como sustituto óseo. Rev Colomb Cienc Quim Farm. 2012;41(1):50-66.

27. Liu C, Huang Y, Shen W, Cui J. Kinetics of hydroxyapatite precipitation at $\mathrm{pH} 10$ to 11 . Biomaterials. 2001;22(4):301-6.

28. Pretto M, Costa AL, Landi E, Tampieri A, Galassi C. Dispersing behavior of hydroxyapatite powders produced by wet-chemical synthesis. J Am Ceram Soc. 2003;86:1534-39.

29. Silverstein RM, Webster FX, Kiemle DJ. "Spectrometric identification of organic compounds". $7 \mathrm{ma}$ ed. New York: John Wiley \& Sons; 2005. 